\title{
La efectividad de una ley de protección al servicio de la conservación de un recurso marino: El ejemplo del camarón fantasma Callichirus major (Decapoda, Callianassidae) de la costa de Brasil
}

\author{
Patricio Hernáez',2 (D), Michael J. Hereman', Camila E. R. Pimenta' (iD, Juliana P. P. Rio', \\ Marcio C. A. João' (1) \& Marcelo A. A. Pinheiro' (1D
}

\begin{abstract}
1.Universidade Estadual Paulista - UNESP, Instituto de Biociências - IB, Grupo de Pesquisa em Biologia de Crustáceos - CRUSTA, Campus do Litoral Paulista, Praça Infante Dom Henrique, s/n , Parque Bitaru, 11330-900 São Vicente, SP, Brasil. (pahernaez@gmail.com; mhereman@gmail.com; camilla.evelyn94@gmail.com; julianario.bio@gmail.com; marcio.camargo96@gmail.com; marcelo.pinheiro@unesp.br)

2. Universidad de Tarapacá - UTA, Facultad de Ciencias, Centro de Estudios Marinos y Limnológicos, Av. General Velásquez, 1775, Arica, Chile.
\end{abstract}

Recibido 18 Junio 2018

Aceptado 03 Diciembre 2018

Publicado 14 Enero 2019

$10.1590 / 1678-4766 e 2019001$

RESUMEN. La mortalidad por pesca ha sido pocas veces estudiada aislando el efecto causado por las capturas. En este estudio se analizó este aspecto usando como modelo biológico al camarón fantasma Callichirus major (Say, 1818), el cual es colectado intensamente en varias regiones de la costa de Brasil. Para ello, la densidad de las galerías y la demografía poblacional de C. major fue analizada en las playas de Gonzaga e Itararé, dos playas contiguas del Estado de São Paulo, Brasil. La playa de Gonzaga fue considerada como control debido a que desde 1992 una ley municipal impide las capturas de C. major, mientras que Itararé fue considerada como tratamiento ya que en este lugar este camarón es recolectado durante todo el año. Los resultados mostraron que la densidad de las galerías y el tamaño de las hembras fueron significativamente mayores en la playa de Gonzaga que en Itararé. En Itararé, la proporción sexual no mostró diferencias significativas de la relación 1:1, lo cual no se ajustó a lo observado en el área sin mortalidad por pesca y al patrón general reportado en otras especies de camarones fantasma del mundo en donde las hembras predominan sobre los machos. El potencial reproductivo fue significativamente menor en Itararé, como efecto del menor número de hembras presentes en esta área sometida a la pesca intensiva de camarones. De acuerdo con una estimativa inicial, un $71 \%$ de los individuos murieron por efecto de la pesca en Itararé. Esto hace inviable el sostenimiento ambiental de $C$. major en esta área dada la actual presión de extracción. La presencia de $C$. major en la playa de Itararé y probablemente en otras áreas próximas donde es capturada, estaría favorecida por el permanente suministro de larvas procedentes de la playa de Gonzaga, en Santos, que está protegida por ley y fiscalización municipal.

PALABRAS-CLAVE. Estructura poblacional, Mortalidad, pesquería artesanal, reproducción, Atlántico Sur Occidental

\begin{abstract}
The effectiveness of a protection law in favor of conservation of an marine resource: The example of the ghost shrimp Callichirus major (Decapoda, Callianassidae) of the coast of Brazil. Studies on mortality due to fishing based on isolating the effect caused by catches are scarce. The present study analyzed this aspect using the ghost shrimp Callichirus major (Say, 1818) as a biological model, which is heavily harvested along the Brazilian coast. For that purpose, density of burrows and population structure of $C$. major was examined at two adjacent beaches, Gonzaga and Itararé, from the State of São Paulo, Brazil. Gonzaga beach was considered as control because since 1992 a municipal law prevents the capture of $C$. major, whereas Itararé as treatment since in this place the species is harvested throughout the year. Burrow density and female CL size were significantly higher in Gonzaga than that Itararé. Sex-ratio did not differ significantly from 1:1 in Itararé, differing from the observed in the area without fishing mortality and also to the general pattern reported in other species of ghost shrimps in which females outnumbered than males. Reproductive potential was significantly smaller in Itarare than Gonzaga, as result of the reduced number of females present in this area relative to Gonzaga. A preliminary estimation indicates that $71 \%$ of shrimps in Itararé died as result of fishing. This information suggests non-viability of C. major in this area given the current pressure of fishing. Presence of $C$. major in the beach of Itararé and probably in other nearby areas where exploitation occurs would be favored by the permanent supply of larvae from Gonzaga beach where the species is protected by and municipal surveillance.
\end{abstract}

KEYWORDS. Population structure, mortality, artisanal fishery, reproduction, south western Atlantic.

El objetivo fundamental de la protección de cualquier recurso biológico es asegurar su continuidad en el ambiente donde este se desarrolla, ya sea para su conservación o para su uso sostenible como fuente comercial o particular. En algunos países como Brasil, la protección de los recursos biológicos es administrada a nivel Federal a través del Instituto Chico Mendes de Conservacão da Biodiversidade (ICMBio) perteneciente al Ministerio de Meio Ambiente
(MMA) con la creación en el ambiente marino de unidades de conservación (UCs) bajo un criterio de protección integral o sustentable. En particular, para el Estado de São Paulo, se crearon tres áreas de protección ambiental (APAs), Norte, Centro y Sur, siendo la APA del litoral centro la mayor de ellas con 450 mil hectáreas (SÃo PAULO, 2008). En esa APA marina, se encuentra la Bahía de Santos que es una área de exclusión de protección debido a la existencia del puerto 
internacional del mismo nombre. No obstante, algunas leyes gubernamentales o municipales pueden asegurar la protección de determinadas poblaciones naturales inclusive en estas áreas no protegidas. Un ejemplo de ello es la ley $\mathrm{n}^{\circ} 850$ promulgada en 1992 por el Municipio de Santos en la cual se prohibió la captura de especimenes del género Callichirus (Callianassidae). Esta medida fue promulgada con base en las observaciones realizadas por S. D. A. Rodrigues (datos no publicados) que resaltaron la necesidad de proteger al camarón excavador Callichirus major (Say, 1818). Dicha ley se mantiene en vigencia hasta la actualidad con la estricta supervisión de la policía municipal que fiscaliza de forma efectiva el cumplimiento de esta ordenanza municipal en la playa de Santos. Ya en la playa contigua, en el municipio de São Vicente, la extracción de $C$. major es completamente permitida. En otras ciudades costeras del Estado de São Paulo (e.g., Praia Grande) y en el Estado de Santa Catarina (e.g., Balneario Camboriú), también existen leyes que impiden la captura y comercialización de C. major. Sin embargo, sólo en las playas de Santos se cumple de forma efectiva esta normativa gracias a la permanente fiscalización de la policía municipal.

Como otras especies de Callichirus [e.g., Callichirus garthi (Retamal, 1975): P. Hernáez, datos no publicados; Callichirus seilacheri (Bott, 1955): HernáEZ \& GrandARodríGuez, 2015], C. major es intensamente capturada por recolectores locales quienes la usan directamente o comercializan como carnada en la pesca deportiva de la costa sureste y sur de Brasil (Borzone \& SouZA, 1996; Rodrigues \& Shimizu, 1997; Souza \& Borzone, 2003). Este camarón es uno de los macroinvertebrados más conspicuos en las playas de arena de la costa del Atlántico Occidental. Su distribución geográfica abarca un amplio intervalo de latitudes a lo largo de la costa Americana $\left(34^{\circ} \mathrm{N}-27^{\circ} \mathrm{S}\right)$. Como se define actualmente, C. major se distribuye desde Beaufort en la costa de Carolina del Norte hasta el sur de la Isla Padre en el noroeste del Golfo de México (HaY \& SHORE, 1918; Felder, 1973; Williams, 1984) y desde Cartagena en el Caribe colombiano a la costa del Estado de Santa Catarina en la región sur de Brasil (Melo, 1999). Callichirus major construye galerías simples compuestas por una sola entrada y habitadas individualmente por un macho o una hembra (PoHL, 1946; Rodrigues \& SHIMIzU, 1997). La abundancia relativa de $C$. major puede alcanzar valores superlativos hasta monopolizar completamente el sustrato en relación a otras especies de camarones fantasma (RodRIGUES \& SHIMIZU, 1997).

Todo este escenario natural y pesquero ofrece una valiosa oportunidad para evaluar si la exclusión de la presión de pesca, como efecto perturbador de las poblaciones naturales (ver TuRNER \& MeYer, 1993), provoca un efecto positivo o negativo en la densidad de las galerías y la demografía poblacional de C. major. Para ello, en el presente estudio se propuso analizar la densidad de las galerías, proporción sexual, el tamaño promedio de ambos sexos, la estructura poblacional y la periodicidad reproductiva del camarón fantasma C. major en las playas de Gonzaga y Itararé, dos playas contiguas del litoral del Estado de São Paulo, Brasil. Una de estas playas (Gonzaga - Municipio de Santos), sin el efecto perturbador antropogénico provocado por las capturas de individuos de la población, mientras que la otra (Itararé Municipio de São Vicente), sometida a un intenso régimen de capturas por parte de recolectores locales.

\section{MATERIALES Y MÉTODOS}

Sitios de estudio. La estimativa de la densidad y la colecta de ejemplares de C. major (Fig. 1) fueron realizadas mensualmente desde mayo de 2015 hasta abril de 2016 en las playas de Gonzaga (Municipio de Santos) e Itararé (Municipio de São Vicente), ambas localizadas en la Bahía de Santos (Fig. 2). Como control se usó a la playa de Gonzaga ya que la ley municipal no 850 prohíbe la captura de C. major, mientras que como tratamiento se usó a la playa de Itararé, en la cual este camarón es capturado permanentemente debido a que no existe ninguna normativa que lo impida. La distancia entre los sitios de colecta es de aproximadamente $5 \mathrm{~km}$, lo cual permitió disminuir a 1 día la toma de información entre las playas. En cuanto a la dinámica de estas playas, ambas han sido clasificadas por Souza (2012) como disipativas debido a la presencia de un tren de oleaje de baja energía, una pendiente de entre $5-6^{\circ}$ y una granulometría que varía de arena fina $(0.25 \mathrm{~mm})$ a muy fina $(0.125 \mathrm{~mm})$. Además, tanto Gonzaga como Itararé reciben el aporte permanente de materia orgánica producto de las aguas domésticas de las ciudades de Santos y São Vicente, que son canalizadas hacia el mar a través de un emisario submarino (Fig. 3).

Densidad poblacional. La densidad poblacional de $C$. major fue estimada a través del recuento del número de galerías por unidad de área. Este método es uno de los más utilizados en la evaluación de la densidad poblacional de camarones fantasma (e.g., HaILSTONE \& STEPHENSON, 1961; Branch \& DaY, 1984; Dumbauld et al., 1996; HernáEZ \& WeHrTMANN, 2007) y resulta eficiente cuando la especie estudiada construye galerías con una entrada, dada la estrecha relación existente entre el número de galerías $y$ el de camarones. La unidad de muestreo utilizada en el recuento de las galerías fue de $1 \times 1 \mathrm{~m}\left(=1 \mathrm{~m}^{2}\right)$, lo que permitió minimizar el producto de la varianza por el costo relativo (S. D. A. Rodrigues; M. Pinheiro, datos no publicados) y consecuentemente obtener un valor representativo del patrón de distribución espacial de C. major. La forma plana o como volcán y el diámetro de las aberturas de las galerías construidas por C. major (2-8 $\mathrm{mm}$ ) facilitó notoriamente su identificación y recuento durante cada actividad de campo. En cada sitio de colecta, fueron implementados tres transectos permanentes $\left(\mathrm{T}_{1}, \mathrm{~T}_{2}, \mathrm{~T}_{3}\right)$ y paralelos a la línea de marea baja $(\sim 0.20 \mathrm{~m})$. Los transectos estuvieron separados entre sí por una distancia de $10 \mathrm{~m}$, siendo $\mathrm{T}_{1}$ el más alejado a la línea de marea y $\mathrm{T}_{3}$ el más cercano a ella (Fig. 3). A continuación, se posicionó cada unidad de muestreo de $1 \mathrm{~m}^{2}$ de forma consecutiva a lo largo de cada transecto por un total de 50 veces. Luego se hizo el recuento de las galerías contenidas 


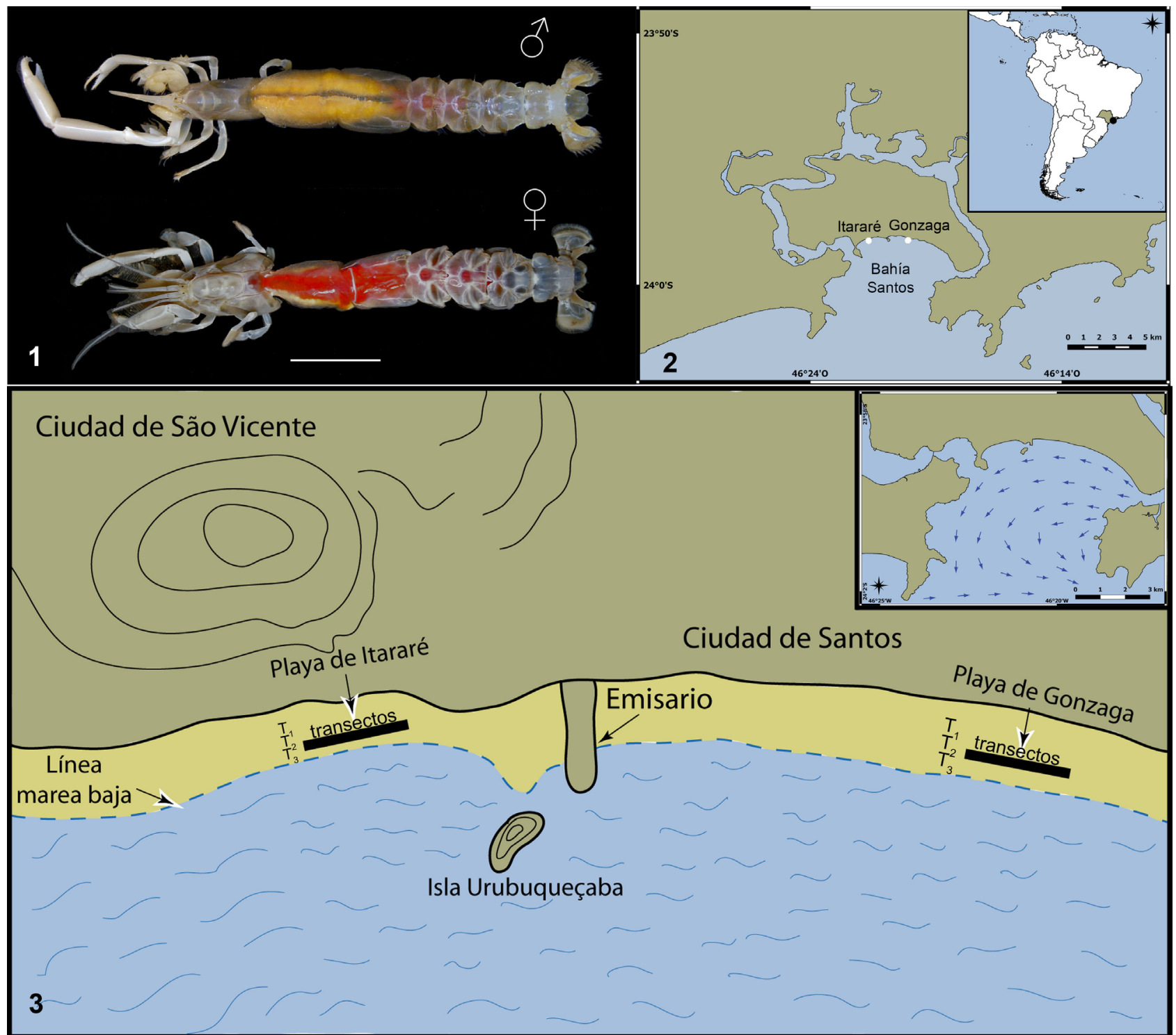

Figs 1-3. Ejemplar macho y hembra del camarón fantasma Callichirus major (Say, 1818), vista dorsal, barra de escala: 1cm (Fig. 1); localización espacial de la playa de Gonzaga y la playa de Itararé en la Bahía de Santos, São Paulo, Brasil (Fig. 2); representación esquemática de los transectos implementados en ambos sitios de estudio, en el recuadro se muestra el patrón de corrientes superficiales de la Bahía de Santos según Harari et al. (2002, 2013) (Fig. 3).

en cada uno de los cuadrantes y se procedió a calcular el promedio de la densidad de galerías de C. major por cada transecto.

Dado que todas las playas de la Bahía de Santos poseen características morfodinámicas similares (SouZA, 2012), se asumió que la abundancia poblacional expresada como la densidad de las galerías de C. major también sea similar entre las playas de esta bahía. Sin embargo, debido a que en Itararé está permitida la captura de C. major, se esperó que exista una disminución del valor de la densidad en comparación con el valor esperado en Gonzaga, debido al efecto negativo de la presión de pesca. Para probar esta hipótesis, la densidad de los transectos fue comparada entre las playas a través de una prueba de Mann-Whitney (U), debido a que los datos no se distribuyeron normalmente (Sokal \& Rohlf, 1995). Cuando se detectaron diferencias significativas entre las playas, se realizó un análisis de regresión GLM (Generalized Linear Models: MCCuLLAGH \& NELDER, 1989) para evaluar el efecto de la variabilidad espacial (transectos) y temporal (meses de colecta) dentro de cada playa sobre la potencial diferencia de la densidad entre ambas playas. El análisis de GLM ofrece una robusta alternativa para el establecimiento de relaciones causa-efecto cuando los errores de los datos no poseen distribuciones normales (e.g., Binomial, Poisson, Gamma, etc.) y las varianzas no son constantes.

Colecta de camarones. Se colectaron ejemplares de C. major en la zona intermareal de la playa de Gonzaga y Itararé. Los ejemplares fueron colectados a la misma altura pero fuera del área donde estuvieron instalados los transectos de manera de no influenciar la estimación mensual de la 
densidad. La colecta de camarones fue realizada durante el periodo de marea baja $(\sim 0.20 \mathrm{~m})$ con la ayuda de una bomba de succión tipo "yabbie" (diámetro $=7.7 \mathrm{~cm}$, largo $=100 \mathrm{~cm}$ ) y haciendo un esfuerzo de muestreo equivalente a tres horas para un total de cuatro personas involucradas en la colecta de camarones. El uso de bombas de succión tipo "yabbie" ha demostrado ser un método eficiente de muestreo de organismos que viven en madrigueras intermareales (S. D. A. Rodrigues, datos no publicados). Durante la actividad de muestreo, cada galería fue bombeada no más de cinco veces. El desmoronamiento del sedimento alrededor de la galería, producido durante la extracción de cada camarón, evitó la reinspección de una misma galería. Cada camarón colectado fue lavado con agua de mar, almacenado de forma individual en una bolsa plástica y congelado a $-2^{\circ} \mathrm{C}$ hasta su análisis en el laboratorio. Parte del material analizado en este estudio fue depositado en la colección carcinológica CRUSTA de la Universidade Estadual de São Paulo, São Vicente, Brasil (CRUSTA 160058).

En el laboratorio, cada camarón fue sexado de acuerdo a la morfología del primer par de pleópodos (machos: bisegmentado; hembras: tri-segmentado - J. P. P. Rio, datos no publicados). Adicionalmente, las hembras fueron clasificadas de acuerdo con la presencia/ausencia de embriones adheridos a los pleópodos (ovígeras y no ovígeras, respectivamente). Alternativamente, en cada camarón se midió la longitud del cefalotórax (LC, desde el margen post-orbital al margen posterior del cefalotórax) con la ayuda de un caliper digital de precisión $(0.05 \mathrm{~mm})$.

Estructura poblacional. La proporción sexual de $C$. major fue calculada en cada playa y comparada con la razón teórica 1:1 propuesta por FISHER (1930) para poblaciones naturales. Alternativamente se verificó si la proporción sexual por clase de tamaño $(1 \mathrm{~mm})$ en Gonzaga y Itararé siguió alguno de los patrones descritos por WeNNER (1972). En ambos casos, el valor obtenido fue testeado a través de la prueba binomial (WILSON \& HARDY, 2002), considerando un mínimo de diez camarones por cada clase de tamaño para garantizar el poder estadístico de la prueba. Cuando esta condición no pudo ser cumplida, los camarones fueron agrupados en una clase de tamaño anterior o posterior hasta completar el mínimo número requerido para correr la prueba.

La remoción selectiva de los individuos más grandes en una población natural provoca una disminución progresiva del tamaño promedio (e.g., LANDE et al., 2003; HUTCHINGS \& Reynolds, 2004; Hutchings \& Baum, 2005; Hsieh et al., 2006; LonghuRst, 2006). Dado esto, se espera que la presión de pesca en Itararé provoque una disminución del tamaño promedio de los machos y las hembras de C. major en comparación con el valor esperado en la playa de Gonzaga. Para probar esta hipótesis, la longitud del cefalotórax (LC) de cada sexo fue comparada entre las playas usando la prueba de Mann-Whitney (U), dado que los datos empíricos no se distribuyeron normalmente (SOKAL \& RoHLF, 1995). El tamaño también fue comparado a nivel de transectos (e.g., machos: Gonzaga $\mathrm{T}_{1} \mathrm{x}$ Itararé $\mathrm{T}_{1}$ ) usando una prueba de
Kruskal-Wallis (KW), dado que las varianzas no fueron homocedásticas (SOKAL \& RoHLF, 1995). De forma adicional, se implementó un análisis de GML para verificar si las diferencias entre la LC de cada sexo se deben a la alteración espacial o temporal de la composición de tallas provocada por una serie de procesos demográficos como el reclutamiento, crecimiento, mortalidad, entre otros, intrínsecos de cada playa. Este último análisis fue realizado solo cuando fueron detectadas diferencias significativas entre las playas.

La estructura poblacional de C. major fue examinada en cada playa a través de la construcción de histogramas de frecuencia en clases de tamaño de $1 \mathrm{~mm}$ LC para machos y hembras. A continuación, las componentes normales de la distribución de tamaños de cada sexo fueron identificadas y separadas por el método de Bhattacharya y posteriormente confirmadas por la rutina de NormSep (ver paquete FiSAT - GaYANILO et al., 1996), con la notación del promedio para cada cohorte. Se espera que el promedio de cada cohorte de Itararé sea menor que el registrado en la playa de Gonzaga dado que la captura selectiva de los individuos de mayor tamaño provoca la disminución del tamaño promedio de los diferentes grupos de edades (HutCHINGS \& REYNOLDS, 2004).

Parámetros reproductivos. La biología reproductiva de C. major fue examinada a través del análisis del tamaño promedio de las hembras ovígeras, el potencial reproductivo y la periodicidad reproductiva de esta especie en cada local de estudio. El tamaño de las hembras ovígeras fue comparado entre las playas por medio de una prueba de Mann-Whitney (U) (SOKAL \& Rohlf, 1995). En el caso de la existencia de diferencias significativas de la LC, se implementó un análisis de GLM para descartar si la potencial diferencia del tamaño de las hembras ovígeras entre las playas es afectada por la variabilidad espacial y temporal intrínseca de cada playa. El potencial reproductivo fue estimado como la proporción de hembras ovígeras con relación al número total de hembras sexualmente maduras y colectadas en cada playa durante el periodo de estudio. En esta ocasión se utilizó como tamaño de mínima madurez sexual la LC de la hembra ovígera más pequeña capturada en Gonzaga $(=11.1 \mathrm{~mm})$ y Itararé $(=13.7 \mathrm{~mm})$. Esta proporción fue comparada entre las playas usando una prueba $Z(\alpha=0.05)$ (SOKAL \& RoHLF, 1995). La periodicidad reproductiva fue estimada para cada playa con base en la variación temporal de la proporción de hembras ovígeras del total de hembras sexualmente maduras y colectadas bimensualmente, dado el bajo número de ejemplares colectados en algunos meses. La sincronización entre la periodicidad reproductiva de las playas fue verificada por medio del coeficiente de correlación de Spearman $(\alpha=0.05)(\mathrm{ZAR}, 2010)$.

\section{RESULTADOS}

Densidad poblacional. Un total de 1800 unidades de muestreo de $1 \mathrm{~m}^{2}$ fueron desplegadas en cada una las playas (Gonzaga y Itararé) durante todo el periodo de estudio. La densidad anual de C. major en la playa de Gonzaga varió 
desde 0 a 18 galerías $/ \mathrm{m}^{2}$ (mediana [rango intercuartilico]: $7[5]$ galerías $/ \mathrm{m}^{2}$ ) y de 0 a 14 galerías $/ \mathrm{m}^{2}$ (2[3] galerías/ $\mathrm{m}^{2}$ ) en Itararé, siendo el valor de la mediana de Gonzaga equivalente a 3.5 veces el valor registrado en Itararé (Fig. 4). Dentro de cada playa, el valor de la densidad mostró diferencias significativas entre los transectos (Gonzaga: $\mathrm{KW}=102.8, \mathrm{P}<0.001$; Itararé: KW=143.1, $\mathrm{P}<0.001)$. En Gonzaga, el máximo valor de la mediana se registró en el transecto intermedio $\left(\mathrm{T}_{2}\right)$, mientras que en Itararé este valor fue registrado en el transecto más cercano a la línea de marea $\left(\mathrm{T}_{3}\right)$. Con excepción del mes de mayo, el valor mensual de la densidad fue siempre mayor en Gonzaga que en Itararé, siendo el coeficiente de variación del área protegida (Gonzaga) menor (CV: $48.9 \%$ ) que el de Itararé (CV: $91.5 \%)$.

Una comparación estadística confirmó la existencia de diferencias significativas en la mediana anual $\left(\mathrm{U}=2.72 \cdot 10^{6}\right.$, $\mathrm{P}<0.001)$ y por transecto $\left(\mathrm{T}_{1}, \mathrm{U}=3.38 \cdot 10^{5}, \mathrm{P}<0.001 ; \mathrm{T}_{2}\right.$, $\left.\mathrm{U}=3.22 \cdot 10^{5}, \mathrm{P}<0.001 ; \mathrm{T}_{3}, \mathrm{U}=2.54 \cdot 10^{5}, \mathrm{P}<0.001\right)$ entre las playas (Fig. 4). El análisis de GLM resaltó la influencia significativa de la variabilidad espacial y temporal del número de las galerías sobre el valor de la densidad anual de C. major en cada playa (Gonzaga: $\mathrm{F}=59.3, \mathrm{P}<0.0001, \mathrm{GL}=1799$; Itararé: $\mathrm{F}=100.9, \mathrm{P}<0.0001, \mathrm{GL}=1799)$. En Gonzaga, el modelo explicó el $54.1 \%$ de la variabilidad observada en la densidad, mientras que en Itararé explicó el $66.7 \%$. La interacción entre la componente espacial (transectos) y temporal (meses) también demostró ser significativa en cada playa (Gonzaga: $\mathrm{F}=47.3, \mathrm{P}<0.0001, \mathrm{GL}=22$; Itararé: $\mathrm{F}=21.7, \mathrm{P}<0.0001, \mathrm{GL}=22$ ).

Estructura poblacional. Un total de 950 ejemplares de $C$. major (Gonzaga: $\mathrm{N}=546$, Itararé: $\mathrm{N}=404$ ) fueron colectados durante todo el periodo de estudio. En la playa de Gonzaga el número capturado de hembras $(\mathrm{N}=342)$ fue significativamente superior al de los machos $(\mathrm{N}=204)$ $\left(\chi^{2}=34.88, \mathrm{P}<0.01\right)$, con $62.3 \%(\mathrm{n}=213)$ de estas hembras con huevos. El tamaño de los machos varió entre 6.9 y 20.1 $\mathrm{mm}$ LC (15.5 [4.0] mm LC) y el de las hembras entre 8.9 y $22.2 \mathrm{~mm}$ LC (17.3 [2.3] mm LC). El tamaño mínimo y máximo de las hembras ovígeras fue de 11.1 y $21.5 \mathrm{~mm} \mathrm{LC}$, respectivamente (17.4 [2.0] mm LC). En la playa de Itararé, el número total de machos y hembras fue estadísticamente similar (M:H, 217: 187; $\left.\chi^{2}=2.23, \mathrm{P}>0.05\right)$, con un total de $53.5 \%(n=100)$ de las hembras ovígeras en este sitio. El tamaño de los machos varió entre 7.3 y $20.7 \mathrm{~mm}$ LC (15.4 [3.0] mm LC) y el de las hembras entre 9.1 y $21.2 \mathrm{~mm}$ LC (16.7 [2.3] mm LC). Las hembras ovígeras midieron entre 13.7 y $21.0 \mathrm{~mm} \mathrm{LC}(17.0$ [1.8] mm LC).

El tamaño (LC) de los machos no difirió significativamente entre las playas tanto a nivel anual como cuando fue comparado por transecto (Fig. 5). En el caso de las hembras, el tamaño registrado en Gonzaga fue significativamente mayor al de Itararé tanto a nivel anual como a nivel de los transectos 1 y 2 (Fig. 6). El análisis de GLM mostró que tales diferencias estadísticas son debido a la existencia de una significativa variabilidad temporal de la
LC de las hembras en las playas de Gonzaga (Mes: $\mathrm{F}=1.83$, $\mathrm{P}<0.05$, GL=11) e Itararé (Mes: $\mathrm{F}=4.34, \mathrm{P}<0.001, \mathrm{GL}=11$ ).

El análisis de progresión modal de Battacharya reveló en ambas playas la existencia de tres cohortes tanto para machos como para hembras (Figs 7-10). Una comparación visual de las cohortes entre ambas playas demostró que una misma cohorte de machos o de hembras siempre alcanzó un tamaño promedio menor en Itararé que en Gonzaga (e.g. cohorte 2 de machos, Gonzaga $=15.1 \mathrm{~mm}$ LC vs Itararé= 14.4 mm LC; Figs 7-10). Los machos fueron más abundantes que las hembras en las clases de tamaño inferiores a $15 \mathrm{~mm}$ y $16 \mathrm{~mm}$ en las playas de Gonzaga e Itararé, respectivamente (Figs 11, 12). Sobre estas marcas de clases, las hembras comenzaron a aumentar gradualmente en número hasta ser casi completamente más abundantes que los machos en las clases de mayor tamaño (Figs 11, 12).

Parámetros reproductivos. El tamaño de las hembras ovígeras de Gonzaga (17.4 [2.0] mm LC) no difirió significativamente $\left(\mathrm{U}=1.16 \cdot 10^{4}, \mathrm{P}>0.05\right)$ del registrado en Itararé (17.0 [1.8] $\mathrm{mm} \mathrm{LC})$. El potencial reproductivo de C. major en Gonzaga (=0.63) no mostró diferencias
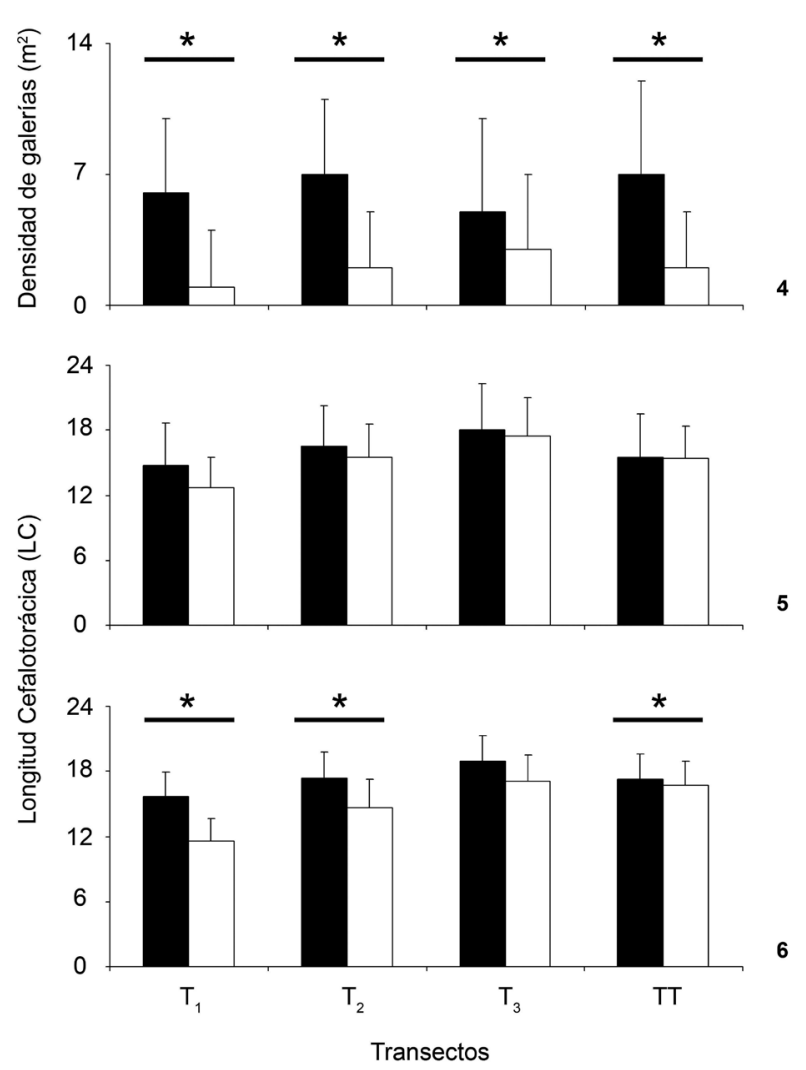

Figs 4-6. Densidad de las galerías del camarón fantasma Callichirus major (Say, 1818) (Fig. 4); mediana de la longitud del cefalotórax (LC) de los machos (Fig. 5); y las hembras (Fig. 6) en las playas de Gonzaga e Itararé. $\mathrm{T}_{1}, \mathrm{~T}_{2} \mathrm{y} \mathrm{T}_{3}$ representan los transectos de muestreo instalados en la zona intermareal y TT el valor total independiente de los transectos de cada playa. Las barras (negras: Gonzaga; blancas: Itararé) representan las medianas, las líneas verticales al rango intercuartílico y los asteriscos a las diferencias significativas entre las medianas de acuerdo a la prueba de Mann-Whitney $(\mathrm{P}<0.05)$. 

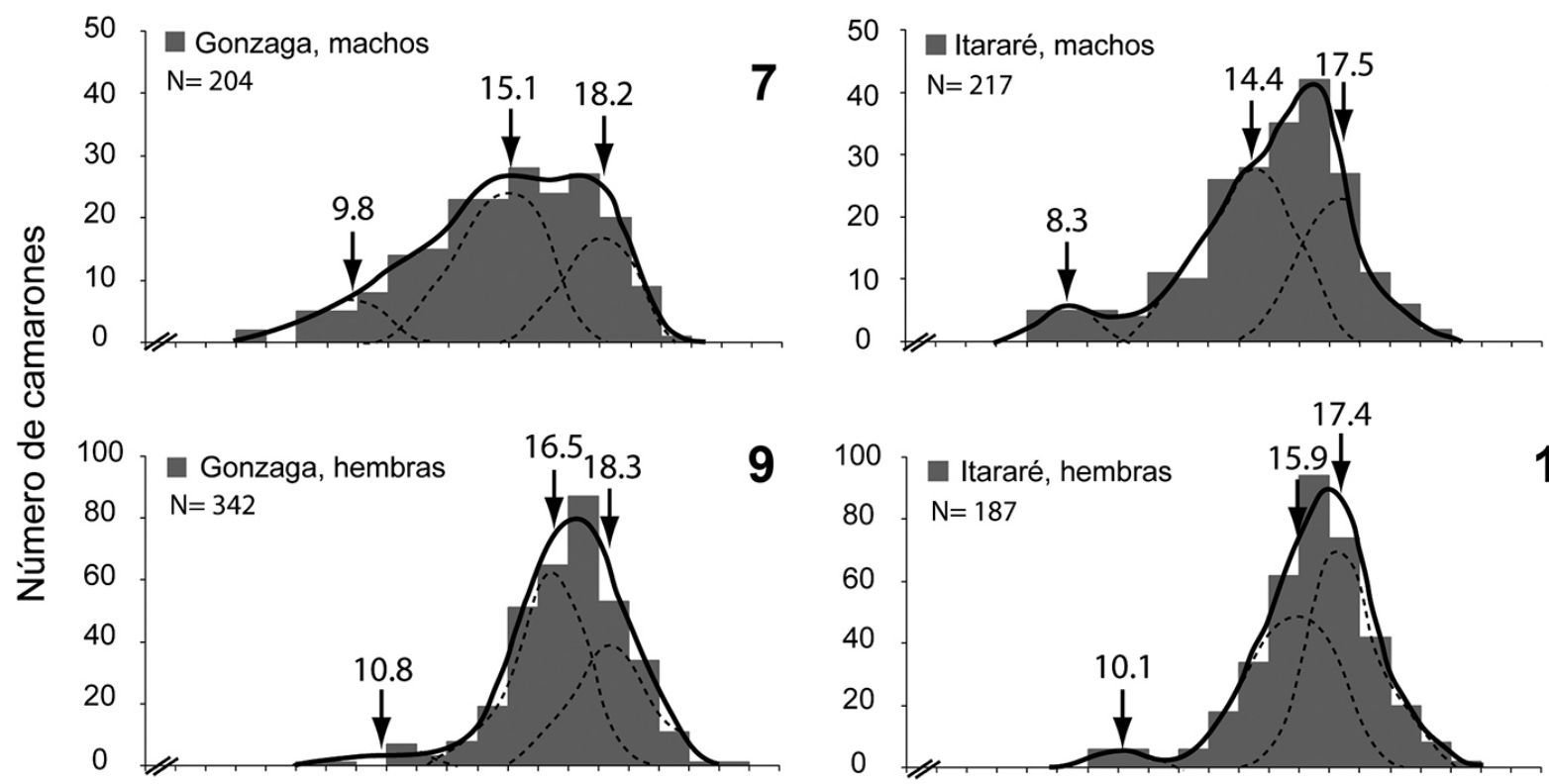

8
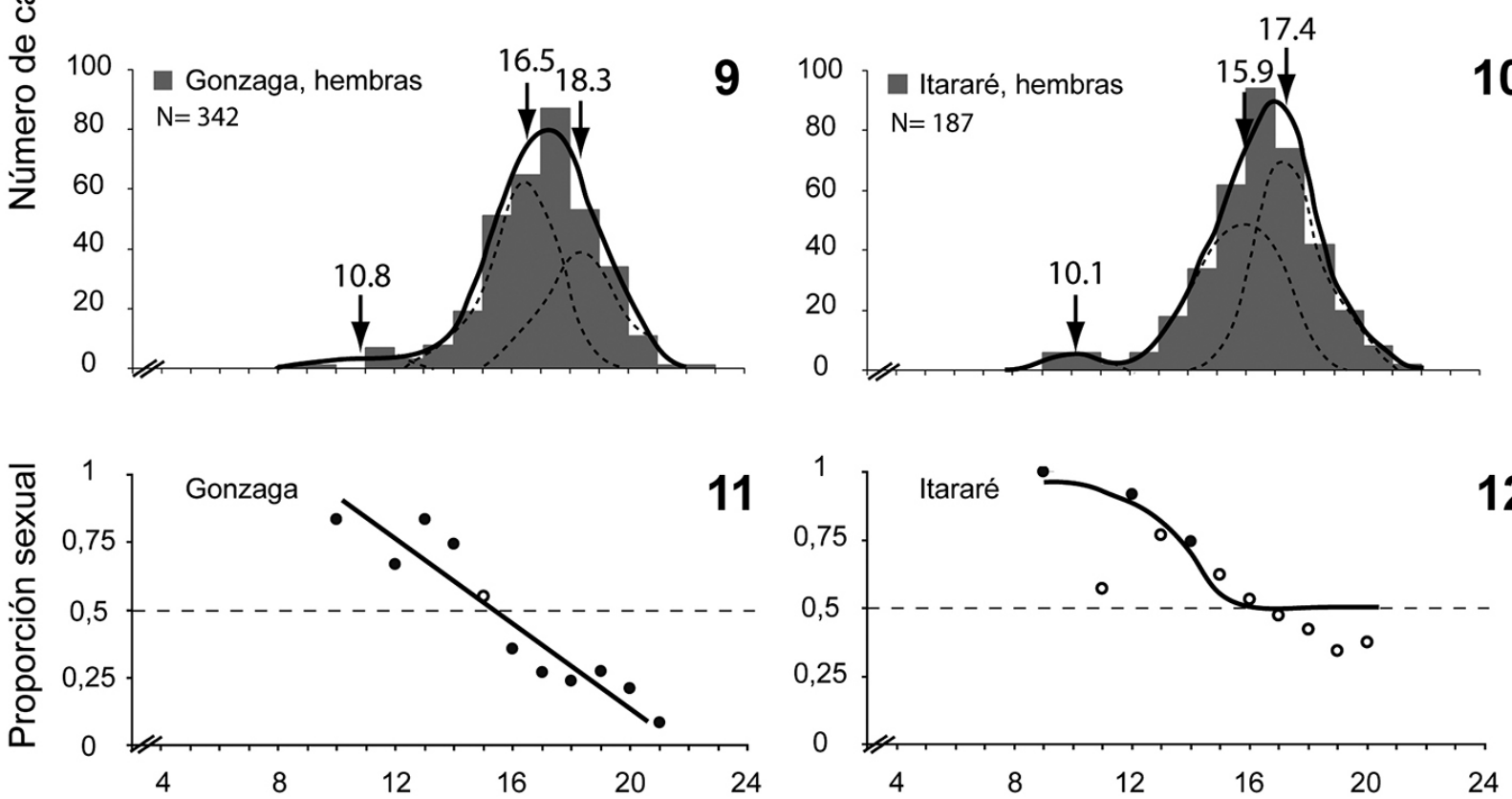

Clases de Tamaño (LC, mm)

Figs 7-12. Distribución de la frecuencia de tamaños de los machos (Figs 7, 8) y las hembras (Figs 9, 10) y proporción sexual (Figs 11, 12) de Callichirus major (Say, 1818) en la playa de Gonzaga e Itararé. La línea segmentada y la continua en Figs 7-10 representan, respectivamente, la estimación de cada cohorte identificada por el método de Bhattacharya y la corrección de Normsep para la estimativa de las cohortes. Las flechas apuntan a la posición del promedio de LC estimada para cada cohorte. Los círculos negros en las Figs 11 y 12 denotan aquellas clases de tamaño en que la proporción sexual fue significativamente diferente de la relación teórica 1:1 $(\mathrm{P}<0.05)$.

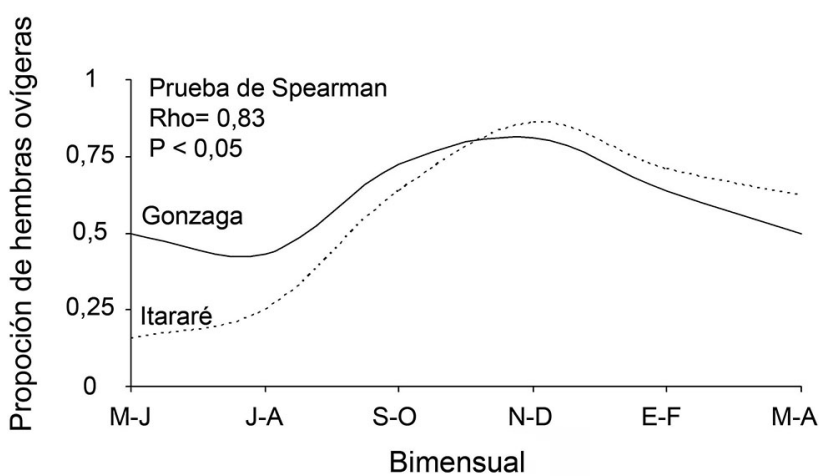

Figura 13. Periodicidad reproductiva en las hembras del camarón fantasma Callichirus major (Say, 1818) en la playa de Gonzaga (línea continua) y Itararé (línea segmentada). significativas con el registrado en la playa de Itararé $(=0.58)$ de acuerdo con la prueba de comparación de las proporciones $(Z=1.06, P>0.05)$. En una escala mensual, siempre hubo presencia de hembras con huevos de $C$. major en cada uno de los sitios de estudio. El coeficiente de variación del porcentaje bimensual de hembras con huevos mostró mayor variabilidad en la playa de Itararé que en Gonzaga ( $46 \%$ vs. $22 \%$, respectivamente). El patrón temporal de la aparición de hembras ovígeras mostró una estrecha y significativa correlación entre las playas (correlación de Spearman, $\mathrm{R}_{\mathrm{s}}=0.84, \mathrm{P}<0.05$, Fig. 13), confirmando que la periodicidad reproductiva de $C$. major en ambas playas presentó un ciclo continuo similar a lo largo del año. La periodicidad reproductiva en C. major, fue continua a través del tiempo; sin embargo su intensidad fue más alta ( $>50 \%)$ desde septiembre a febrero en relación a los demás meses. 


\section{DISCUSIÓN}

Desde hace más de dos décadas que no existe presión de extracción sobre C. major en la playa de Gonzaga. Esto se ve reflejado en el aumento que se observa al comparar el valor de la densidad de las galerías registrado en el presente estudio ( $=7$ galerías $/ \mathrm{m}^{2}$ ) con el reportado por RoDRIGUES \& SHimizu (1997) hace aproximadamente 20 años atrás (2.9 galerías $/ \mathrm{m}^{2}$ ). Esta comparación evidencia un aumento aproximado de 2.4 veces la cantidad de galerías por unidad de área desde que las capturas de C. major fueron suspendidas en el municipio de Santos, lo cual resalta la efectividad de la ley que impide las capturas de este camarón. Por otro lado, cada playa mostró una significativa variación de la densidad tanto a nivel espacial como temporal lo que indirectamente demuestra la constante modificación de la estructura poblacional por procesos como el asentamiento de reclutas y/o la muerte de individuos por causas naturales (i.e., depredación, enfermedades, etc.) o no naturales (i.e., mortalidad por pesca) que estaría ocurriendo de forma distinta en ambas playas. Este último proceso solo presente en la playa de Itararé. Efectivamente, el valor de la densidad en la playa de Itararé $\left(=2\right.$ galerías $\left./ \mathrm{m}^{2}\right)$ fue 3.5 veces menor al registrado en la playa de Gonzaga $\left(=7\right.$ galerías $\left./ \mathrm{m}^{2}\right)$ lo que coloca de manifiesto el efecto negativo que genera la presión de pesca sobre la densidad de C. major en la playa de Itararé. Considerando que la densidad de galerías en Gonzaga representa el valor que tendrá esta variable en una área en donde la única causa de muerte es la natural, la diferencia entre la densidad de Gonzaga e Itararé dará una estimación aproximada de los individuos que son muertos por pesca. Siguiendo esta línea de razonamiento, se estimó que el $71 \%$ de los individuos son muertos por pesca en la playa de Itararé. Esta información es relevante ya que se sugiere que la mortalidad por pesca en cualquier población natural sometida a un esfuerzo de captura debe ser mantenida abajo del 50\% del total de las muertes (SPARRE \& Venema, 1997). Cuando este valor es superado, la reproducción y el reclutamiento se ven afectados de forma permanente colocando en riesgo la supervivencia de cualquier población local (Cushing, 1968; Gulland, 1971).

En general, las especies de camarones fantasma exhiben una proporción sexual sesgada hacia las hembras (Callichirus garthi: Hernáez \& Wehrtmann, 2007; Lepidophthalmus louisianensis: FELDER \& LOVETT, 1989; Lepidophthalmus sinuensis: NATES \& FeLDER, 1999; Sergio mirim: Pezzuto, 1998; Trypaea australiensis: BUtLER et al., 2009). Esta tendencia fue confirmada por nuestras observaciones en Gonzaga y también coincide con el patrón general reportado para C. major procedente de otras localidades de la costa brasilera (ver BOTTER-CARVALHO et al., 2007; AlvEs-Junior et al., 2013). De acuerdo con la teoría de asignación sexual de FisHER (1930), la selección natural favorece la existencia de una descendencia compuesta por un igual número de machos y hembras (1:1). Una proporción sexual sesgada hacia las hembras puede estar asociada a la existencia de diferencias entre la tasa de mortalidad de cada sexo durante la etapa larval o postlarval de estas especies. En favor de esta línea de razonamiento, el análisis de la proporción sexual por clases de tamaño de C. major en Gonzaga mostró que la clase de $15 \mathrm{~mm}$ es la única en que la proporción sexual no fue sesgada hacia uno de los dos sexos. Bajo y sobre esta marca de clase, los machos y las hembras alternaron, respectivamente, la dominancia en la proporción de sexos. Esta información sugiere la existencia de una alta tasa de mortalidad de las hembras en la etapa larval de C. major, lo cual generó un sesgo hacia los machos en las marcas de clases inferiores a $15 \mathrm{~mm}$. Por el contrario, la mortalidad fue selectiva hacia los machos por sobre esta marca de clase, generando un sesgo hacia las hembras en todas las marcas de clases superiores a $15 \mathrm{~mm}$.

En cambio en Itararé, resultó interesante observar que sobre la marca de clase de $15 \mathrm{~mm}$ e incluso en algunas marcas de clase inferiores, la proporción sexual no mostró diferencias significativas de la relación 1:1. Este aspecto sugiere que la presión de pesca que es exclusiva de Itararé puede afectar selectivamente a las hembras, generando una reducción del número de hembras en relación al de los machos y consecuentemente provocar un cambio en el estado natural de la proporción de sexos (usualmente sesgada para hembras) en la mayor parte de las marcas de clases que componen la estructura poblacional de C. major en esta playa. De acuerdo con WENNER (1972), una alta tasa de mortalidad puede afectar de forma selectiva a los individuos de un sexo debido a las diferentes capacidades de escape desarrolladas por los machos y las hembras. Esto podría aumentar considerablemente la probabilidad de ser capturado y por ende afectar la proporción de los sexos en relación a su expresión natural en áreas no explotadas comercialmente como la de Gonzaga. Esta suposición es realizada sobre la base de que $C$. major en Itararé se encuentra sometida a una considerable presión de pesca y por lo tanto, la proporción sexual en este local es el reflejo del estado de este parámetro poblacional bajo un régimen de explotación pesquera.

Otro aspecto importante a destacar es que la distribución de la proporción sexual por clases de tamaño de C. major en Gonzaga se ajustó al patrón de reversión sexual descrito por WENNER (1972) para algunas especies de camarones marinos [e.g. Pandalus jordani Rathbun, 1902, Solenocera membranacea (Risso, 1816)]. Estudios recientes indican que los machos de C. major poseen gónadas con una sección testicular y ovárica (SouzA et al., 2017), mientras que las hembras presentan aberturas genitales extras sobre las coxas del quinto par de pereiópodos, tal cual como sucede en los machos (J. P. P. Rio, datos no publicados). Estos antecedentes, sugieren que la distribución de la proporción sexual por la clase de tamaño de $C$. major observada en la playa de Gonzaga podría esconder una estrategia de cambio de sexo, tal como ocurre en muchos camarones Caridea (BAUER, 2004). Otros estudios son necesarios para profundizar en esta última materia.

La pesca generalmente busca la captura selectiva de los individuos de mayor tamaño (LANDE et al., 2003). Esta selectividad genera una disminución en el tamaño 
promedio individual de la estructura poblacional en las poblaciones comercialmente explotadas (ANDERSON et al., 2008). Nuestros resultados confirmaron parcialmente este supuesto. El tamaño de las hembras fue menor en la playa de Itararé que en Gonzaga, demostrando así el efecto negativo que produce la captura selectiva de los individuos de mayor tamaño sobre la mediana de un sexo. En el caso de los machos, la falta de una diferencia significativa entre las playas tanto a nivel de los transectos como en el total, parece estar relacionada con la selectividad del arte de pesca que concentra el esfuerzo de la captura principalmente sobre las hembras, lo cual minimiza las diferencias entre las medianas de ambas playas. En resumen, la pesca en Itararé parece ser selectiva e incluso podría no sólo ser selectiva hacia los individuos más grandes sino que también hacia los individuos de un mismo sexo. En este caso particular, afectar de forma selectiva a las hembras.

La comparación de los parámetros reproductivos entre ambas playas, mostró la existencia de similitudes en todos los aspectos analizados. De acuerdo con ANDERSON et al. (2008), la selectividad del arte de pesca provoca la eliminación de los individuos de mayor tamaño, afectando así el tamaño promedio de cada sexo. Esto se vio reflejado solo en la disminución significativa del tamaño de las hembras totales pero no en el de las hembras ovígeras en Itararé en comparación con el valor registrado en Gonzaga. El ciclo reproductivo de $C$. major en Itararé estuvo fuertemente sincronizado con el de Gonzaga, siendo ambos caracterizados por un ritmo continuo de reproducción tal como se ha reportado en otras poblaciones de C. major de la costa noreste de Brasil (BotTer-CARVALHo et al., 2007). Este patrón continuo de reproducción podría estar asociado a las condiciones oceanográficas de la Bahía de Santos que al ser un gran estuario, recibe el aporte permanente de las aguas cargadas de nutrientes procedentes de los diferentes canales que componen esta bahía (HARARI et al., 2002). Esta idea ya había sido propuesta por RodRigues \& SHIMIZU (1997) quienes también reportaron la presencia constante de hembras ovígeras para C. major de la Bahía de Santos, argumentando que la reproducción de esta especie fue estacional en otros locales sin el aporte permanente de materia orgánica como la Bahía de São Sebastião (R. M. Shimizu, datos no publicados) y la playa de Atami (Souza et al., 1998) en la región sureste y sur de Brasil, respectivamente. Callichirus major es una especie de hábitos alimenticios generalistas (S. D. A. Rodrigues, datos no publicados), por lo tanto es perfectamente plausible que la reproducción de esta especie se adapte de forma flexible a las condiciones ambientales de cada local. La presencia de un emisario submarino cuya salida está localizada a $4 \mathrm{~km}$, i.e., casi en la boca de la Bahía de Santos (CetesB, 1997), podría también tener un papel preponderante en el suministro de materia orgánica hacia las playas de Gonzaga e Itararé dado el patrón oceanográfico de Bahía de Santos (Harari et al., 2002, 2013).

Con base en la información obtenida en este estudio, el presente trabajo proporcionó evidencias de como la mortalidad por pesca provoca un efecto negativo sobre algunos aspectos de la historia de vida de C. major. Entre los principales parámetros poblacionales que se ven afectados por la presión de pesca están la densidad poblacional, el tamaño de los individuos y la proporción sexual. La presencia de C. major en la playa de Itararé parece estar estrechamente ligada al permanente suministro de larvas procedentes de la playa de Gonzaga. Este suministro podría ser favorecido por el particular sistema de corrientes de la Bahía de Santos que transporta las aguas superficiales en sentido sur-oeste durante marea de Sicigia y de Cuadratura (HARARI et al., 2002, 2013). Basado en esto, se sugiere que la ley que prohíbe las capturas de $C$. major en la playa de Gonzaga sea mantenida en vigencia para asegurar la manutención de la densidad poblacional de esta especie en otras áreas de la Bahía de Santos.

Agradecimientos. Los autores agradecen la Fundação de Amparo à Pesquisa do Estado de São Paulo (FAPESP) por otorgar ayuda financiera a $\mathrm{PH}$ para el desarrollo de su proyecto de post-doctorado (\# 2015/09020-0) y al Conselho de Desenvolvimento Científico e Tecnológico (CNPq) por la beca de investigación otorgada a MAAP (\#305714/2015-5). Los autores agradecen profundamente la esmerada labor de dos revisores anónimos cuyos comentarios contribuyeron de forma sustancial a mejorar la calidad de este manuscrito. Los datos del presente proyecto fueron obtenidos con permiso oficial de colecta otorgado por el Instit uto Brasilero de Recursos Naturais y del Medioambiente (SISBIO/IBAMA-MMA) al segundo autor (\# 47387-2).

\section{REFERENCIAS}

Alves-Júnior, F. A.; Araúuo, M. S. L. C. \& Feitosa, F. A. N. 2013. Crescimento Alométrico de Callichirus major (SAY 1818) (Crustacea: Callianassidae) em uma praia arenosa do Nordeste brasileiro. Tropical Oceanography 42:13-21.

Anderson, C. N. K.; Hsieh, C.-H.; Sandin, S. A.; Hewitt, R.; Hollowed, A.; Beddington, J.; May, R. M. \& Sugihara, G. 2008. Why fishing magnifies fluctuations in fish abundance. Nature 452:835-839.

BAUER, R.T. 2004. Remarkable shrimps. Norman, Oklahoma University Press. 289p.

Borzone, C. \& SouzA, J. R. B. 1996. A extração de corrupto, Callichirus major (Decapoda: Callianassidae), para uso como isca em praias do litoral do Paraná: características da pesca. Nerítica 10:69-79.

Botter-Carvalho, M. L.; Santos, P. J. P. \& Carvalho, P. V. V. C. 2007. Population dynamics of Callichirus major (Say, 1818) (Crustacea, Thalassinidea) on a beach in northeastern Brazil. Estuarine, Coastal and Shelf Science 71(3-4):508-516.

Branch, G. M. \& DAY, J. A. 1984. Ecology of southern African estuaries: Part XIII. The Palmiet River estuary in the south-western Cape. African Zoology 19(2):63-77.

Butler, S. N.; ReID, M. \& Bird, F. L. 2009. Population biology of the ghost shrimps, Trypaea australiensis and Biffarius arenosus (Decapoda: Thalassinidea), in Western Port, Victoria. Memoirs of Museum Victoria 6:43-59.

CETESB. 1997. Relatório de balneabilidade das praias paulistas. São Paulo, Cetesb. 187p.

Cushing, D. H. 1968. Direct estimation of a fish population acoustically. Journal of the Fisheries Board of Canada 25(11):2349-2364.

Dumbauld, B. R.; Armstrong, D. A. \& Feldman, K. L. 1996. Life-history characteristics of two sympatric thalassinidean shrimps, Neotrypaea californiensis and Upogebia pugettensis, with implications for oyster culture. Journal of Crustacean Biology 16(4):689-708.

FELDER, D. L. 1973. An annotated key to crabs and lobsters (Decapoda, Reptantia) from coastal waters of the northwestern Gulf of Mexico. Baton Rouge, Louisiana State University Press. 103p.

Felder, D. L. \& LovetT, D. L. 1989. Application of regression techniques to studies of relative growth in crustaceans. Journal of Crustacean Biology 9(4):529-539. 
FISHER, R. A. 1930. The Genetical Theory of Natural Selection. Claredon, Oxford University Press. 308p.

Gayanilo, F. C.; Sparre, P. \& Pauly, D. 1996. The FAO-ICLARM Stock Assessment Tools (FISAT) User's guide. Rome, Food \& Agriculture Org (FAO). 23p.

GuLLAND, J. A. 1971. Science and fishery management. ICES Journal of Marine Science 33(3):471-477.

Hailstone, T. S. \& Stephenson, W. 1961. The biology of Callianassa (Trypaea) australiensis Dana, 1852 (Crustacea, Thalassinidea). University of Queensland Papers 1(12):259-285.

Harari, J.; CAMARGO, R. \& Miranda, L. B. 2002. Modelagem numérica hidrodinâmica tridimensional da região costeira e estuarina de São Vicente e Santos (SP) - Pesquisa Naval. Revista Marítima Brasileira 15:79-97.

Harari, J.; Ferreira, F. R.; Degaspari, F. A. \& Sartor, S. M. 2013. Modelagem numérica da hidrodinâmica e da dispersão de esgoto na Baía de Santos, SP. Revista Brasileira de Recursos Hídricos 18:205-214.

HAY, W. P. \& ShORE, C. A. 1918. The decapod crustaceans of Beaufort, N. C., and the surrounding region. Bulletin of the United States Bureau of Fisheries 35:369-475.

Hernáez, P. \& Granda-Rodríguez, H. 2015. La comunidad de Mata de Limón, Pacífico central de Costa Rica y la extracción de colonchos Callichirus seilacheri (Bott, 1955) (Decapoda: Axiidea: Callianassidae). Latin American Journal of Aquatic Research 43(3):575-580.

Hernáez, P. \& WehrTmann, I. S. 2007. Population biology of the burrowing shrimp Callichirus seilacheri (Decapoda: Callianassidae) in northern Chile. Revista de Biología Tropical 55(1):141-152.

Hsieh, C. H.; Reiss, C. S.; Hunter, J. R.; Beddington, J. R.; May, R. M. \& Sugihara, G. 2006. Fishing elevates variability in the abundance of exploited species. Nature 443(7113):859.

Hutchings, J. A. \& BAUM, J. K. 2005. Measuring marine fish biodiversity: temporal changes in abundance, life history and demography. Philosophical Transactions of the Royal Society of London B: Biological Sciences 360(1454):315-338.

HutChingS, J. A. \& ReYNOLDS, J. D. 2004. Marine fish population collapses: consequences for recovery and extinction risk. BioScience 13:297-309.

Lande, R.; Engen, S. \& Saether, B. E. 2003. Stochastic population dynamics in ecology and conservation. New York, Oxford University Press. 209p.

Longhurst, A. 2006. The sustainability myth. Fisheries Research 81(2-3): 107-112.

McCullagh, P. \& Nelder, J. A. 1989. Generalized linear models. Boca Raton, CRC Press. 532p.

Melo, G. D. 1999. Manual de identificação dos crustácea decapoda do litoral brasileiro: Anomura, Thalassinidea, Palinuridea, Astacidea. São Paulo, Editora Plêiade, 545p.

NAtes, S. F. \& Felder, D. L. 1999. Growth and maturation of the ghost shrimp Lepidophthalmus sinuensis Lemaitre and Rodrigues, 1991
(Crustacea, Decapoda, Callianassidae), a burrowing pest in penaeid shrimp culture ponds. Fisheries Bulletin 97:526-541.

Pezzuto, P. R. 1998. Population dynamics of Sergio mirim (Rodrigues 1971) (Decapoda: Thalassinidea: Callianassidae) in Cassino Beach, Southern Brazil. Marine Ecology 19(2):89-109.

PoHL, M. E. 1946. Ecological observations on Callianassa major Say at Beaufort, North Carolina. Ecology 27(1):71-80.

Rodrigues, S. D. A. \&. Shimizu, R. M. 1997. Autoecologia de Callichirus major (Say, 1818). Oecologia Brasiliensis 3:155-170.

São PAULO. 2008. Decreto Estadual n ${ }^{\circ} 33.526$, de 8/10/2008, que cria a Área de Proteção Ambiental Marinha do Litoral Centro, e dá providencias correlatas. Diário Oficial do Estado (DOE-I) - Executivo (9/10/2008), p. 5.

SOKAL, R. R. \& RoHLF, F. J. 1995. Biometry: the principle and practice of statistics in biological research. New York, W. H. Freeman and Company. 887p.

Souza, C. R. G. 2012. Praias arenosas oceânicas do estado de São Paulo (Brasil): síntese dos conhecimentos sobre morfodinâmica, sedimentologia, transporte costeiro e erosão costeira. Revista do Departamento de Geografia 1:308-371.

Souza, J. R. B. \& Borzone, C. A. 2003. A extração de corrupto, Callichirus major (Say) (Crustacea, Thalassinidea), para uso como isca em praias do litoral do Paraná: as populações exploradas. Revista Brasileira de Zoologia 20(4):625-630.

Souza, J. R. B.; Borzone, C. A. \& BreY, T. 1998. Population dynamics and secondary production of Callichirus major (Crustacea: Thalassinidea) on a southern Brazilian sandy beach. Archive of Fishery and Marine Research 46(2): 151-164.

Souza, T. L.; Braga, A. A.; López-Greco, L. S. \& Nunes, E. T. 2017. Functional morphology of the male reproductive system in Callichirus major (Crustacea: Decapoda: Axiidea): Evidence of oocytes in the gonad. Acta Zoologica 119:1-10.

SPARRE, P. \& VENEMA, S. C. 1997. Introdução à avaliação de mananciais de peixes tropicais. Parte I: Manual. FAO Documento Técnico sobra as Pescas. Rome, Food and Agriculture Org (FAO). 404p.

Turner, B. L. \& MeYer, W. B. 1993. Environmental change: the human factor. In: McDonnell, J. \& Pickett, S. T. A. eds. Humans as components of ecosystems. New York, Springer, p.40-50.

Wenner, A. 1972. Sex ratio as a function of size in marine Crustacea. The American Naturalist 106:321-350.

WiLliams, A.B. 1984. Shrimps, lobsters, and crabs of the Atlantic coast of the eastern United States, Maine to Florida. Washington, Smithsonian Institution Press. 550p.

WILSON, K. \& HARDY, I. C. 2002. Statistical analysis of sex ratios: an introduction. Sex ratios: concepts and research methods 1:48-92.

ZAR, J.H. 2010. Biostatistical analysis. New Jersey, Prentice Hall. 663p. 